\title{
Experimental and numerical study of wall layer development in a tribocharged fluidized bed
}

\author{
Petteri Sippola ${ }^{1} \dagger$, Jari Kolehmainen ${ }^{2}$, Ali Ozel ${ }^{3}, \mathrm{Xiaoyu} \mathrm{Liu}^{2}$, \\ Pentti Saarenrinne ${ }^{1}$ and Sankaran Sundaresan ${ }^{2}$ \\ ${ }^{1}$ Department of Mechanical Engineering and Industrial Systems, \\ Tampere University of Technology, Tampere, 33720 Finland \\ ${ }^{2}$ Department of Chemical and Biological Engineering, Princeton University, Princeton, NJ 08544, USA \\ ${ }^{3}$ School of Engineering and Physical Sciences, Heriot-Watt University, Edinburgh EH14 4AS, UK
}

(Received 14 December 2017; revised 12 March 2018; accepted 14 May 2018; first published online 26 June 2018)

The effects of triboelectricity in a small-scale fluidized bed of polyethylene particles were investigated by imaging the particle layer in the vicinity of the column wall and by measuring the pressure drop across the bed. The average charge on the particles was altered by changing the relative humidity of the gas. A triboelectric charging model coupled with a computational fluid dynamics-discrete element method (CFDDEM) model was utilized to simulate gas-particle flow in the bed. The electrostatic forces were evaluated based on a particle-particle particle-mesh method, accounting for the surface charge on the insulating walls. It was found that simulations with fixed and uniform charge distribution among the particles capture remarkably well both the agglomeration of the particles on the wall and the associated decrease in the pressure drop across the bed. With a dynamic tribocharging model, the charging rate had to be accelerated to render the computations affordable. Such simulations with an artificial acceleration significantly over-predict charge segregation and the wall becomes rapidly sheeted with a single layer of strongly charged particles.

Key words: fluidized beds, multiphase and particle-laden flows

\section{Introduction}

When two solid surfaces are brought into contact and separated, a net charge transfer may occur between the surfaces. This phenomenon is often referred to as 'triboelectric charging' or simply as 'tribocharging', although no frictional contact is required for charge transfer (Harper 1967). The exact nature of triboelectric charge transfer between insulating surfaces is still not completely understood. There has been a long-lasting debate on whether ions or electrons are responsible for triboelectric charging (McCarty \& Whitesides 2008; Knorr 2011; Lacks \& Sankaran 2011). Furthermore, tribocharging is generally affected by numerous factors such

$\dagger$ Email address for correspondence: psippola@gmail.com 
as impurities and oxides, surface roughness, object geometry and relative humidity, which pose challenges in numerical modelling (Castle 1997; Karner et al. 2014; Schella, Herminghaus \& Schroter 2017).

Most attempts to model triboelectric charging are based on the concept of effective work function difference or contact potential difference between surfaces (Harper 1967; Matsuyama \& Yamamoto 1995; Tanoue et al. 2001; Laurentie, Traoré \& Dascalescu 2013; Korevaar et al. 2014; Mizutani, Yasuda \& Matsusaka 2015; Grosshans \& Papalexandris 2016a,b), which can be regarded as the high-density limit of the surface state theory (Lowell \& Rose-Innes 1980). In short, it is assumed that charge is transferred until the resulting electric potential difference between the surfaces balances out the driving potential or the work function difference. A relatively simple condenser model is commonly used to determine the electric potential difference due to the transferred charge, usually by treating the contact areas as uniformly charged parallel plates (Matsusaka et al. 2010).

Other modelling approaches found in the literature include particle polarization based charging (Pähtz, Herrmann \& Shinbrot 2010; Siu et al. 2014; Yoshimatsu et al. 2016, 2017); using particle electron surface density (Duff \& Lacks 2008); high and low energy electrons (Kok \& Lacks 2009); particle temperature variation based charging ( $\mathrm{Gu}$ et al. 2013); and saturation charge density of the surface limited by dielectric breakdown (Korevaar et al. 2014). These other approaches have been mostly targeted at explaining triboelectricity between particles made of the same material, and therefore are not applicable to cases where different materials are present.

Laurentie et al. (2010, 2013) developed a numerical model compatible with the discrete element method (DEM) to describe the charge transfer between insulating particles. The model is based on the effective work function difference between materials and takes into account the electric field at the contact point, which has been shown to impact insulator triboelectrification (Zhou et al. 2014; Mizutani et al. 2015). The model has also showed good agreement with experimental results in vibrated bed experiments (Laurentie et al. 2010, 2013; Kolehmainen et al. 2017b); in a granular hopper chute flow (Naik et al. 2015); and in granular binary mixtures (Naik et al. 2016).

In gas-solid and granular flows, triboelectric charging may cause particle agglomeration (LaMarche et al. 2010; Lee et al. 2015); affects particle entrainment rate (Fotovat et al. 2017a; Fotovat, Grace \& Bi 2017c; Yang et al. 2017); affects bubble size and shape in bubbling fluidized beds (Jalalinejad, Bi \& Grace 2012; Rokkam et al. 2013); and leads to adhesion of particles to the walls (Hendrickson 2006; Sowinski, Mayne \& Mehrani 2012; Salama et al. 2013; Song \& Mehrani 2017). For instance, this may result in wall sheeting seen in polymerization reactors that hinders the reactor performance (Hendrickson 2006).

While it is generally agreed that electrostatic forces cause wall sheeting, the exact mechanism still remains under debate, and it is unclear how the electrostatic forces lead to multiple particles thick layers. Mehrani and co-workers (Salama et al. 2013; Song \& Mehrani 2017) have suggested that the particle sheet on the wall would consist of alternating layers of bipolar particles that attract each other. While this mechanism is feasible, it requires a fairly wide charge distribution. Furthermore, it has been shown by Lee et al. (2015) that nearly monodisperse particles exhibit a fairly narrow charge distribution, making this mechanism unlikely for monodisperse particles. Dielectrophoresis has been shown to affect particle agglomeration (LaMarche et al. 2010; Liu et al. 2010; Lee et al. 2015; Siu et al. 2015) and might also play an important role in wall sheeting. 
In the present study, the effects of triboelectric charging in a small fluidized bed of polyethylene particles were investigated both experimentally and numerically. The focus is on particle-wall adhesion, which was found to be a considerable side effect of triboelectric charging in the present experiments. We investigated the wall sheeting indirectly by measuring the pressure drop across the fluidized bed and directly by particle image velocimetry (Westerweel, Dabiri \& Gharib 1997). The magnitude of average particle charge was adjusted by changing the relative humidity (RH, 0-60\%) while keeping other parameters constant. The triboelectric charging model proposed by Laurentie et al. (2013) was employed to describe triboelectric charging in the simulated bed, and effective work functions required by the model were calibrated by measuring the mean charge of particles in the bed by a Faraday cup technique (Fotovat, Bi \& Grace 2017b). The numerical results were compared with experimental data to investigate possible modelling problems encountered when the model is used to predict fluidization behaviour.

\section{Mathematical modelling}

The coupled motion of the gas phase and the solid particles is solved in terms of computational fluid dynamics (CFD) and discrete element method via an OpenFOAM (Jasak et al. 2007)-based solver. The underlying equations of the CFD-DEM model are briefly presented before discussing the triboelectric charging model.

According to the soft-sphere contact model (Cundall \& Strack 1979), particle motion is tracked by solving Newton's equations of motion of individual particles:

$$
\begin{gathered}
m_{i} \frac{\mathrm{d} \boldsymbol{v}_{i}}{\mathrm{~d} t}=\sum_{j}\left(\boldsymbol{f}_{c, i j}^{n}+\boldsymbol{f}_{c, i j}^{t}\right)+\boldsymbol{f}_{e, i}+\boldsymbol{f}_{g \rightarrow p, i}+m_{i} \boldsymbol{g} \\
I_{i} \frac{\mathrm{d} \boldsymbol{\omega}_{i}}{\mathrm{~d} t}=\sum_{j} \boldsymbol{T}_{i j},
\end{gathered}
$$

where $m_{i}, I_{i}, \boldsymbol{v}_{i}$ and $\omega_{i}$ are the mass, the moment of inertia, the translational velocity and the angular velocity of particle $i$, respectively. The forces acting on particle $i$ are classified as the normal and tangential contact forces $\left(\boldsymbol{f}_{c, i j}^{n}\right.$ and $\boldsymbol{f}_{c, i j}^{t}$, respectively) due to other particles and walls, the electrostatic force $\left(\boldsymbol{f}_{e, i}\right)$, the force due to surrounding gas $\left(\boldsymbol{f}_{g \rightarrow p, i}\right)$ and the gravitational force $\left(m_{i} \boldsymbol{g}\right)$. Torque acting on particle $i$ due to particle $j$ is $\boldsymbol{T}_{i j}=\boldsymbol{R}_{c, i j} \times \boldsymbol{f}_{c, i}$, where $\boldsymbol{R}_{c, i j}$ is a vector from the centre of particle $i$ to the contact point. Rolling friction and torque due to forces other than contact forces are not accounted in this study.

The equations describing the contact forces on a particle are given in table 1 , where $\delta_{n}$ and $\delta_{t}$ are the normal and the tangential overlap distance of the particles, respectively; $\boldsymbol{n}_{i j}$ is an unit vector pointing from particle $j$ to particle $i$ and $\mu_{s}$ is the sliding friction coefficient. The tangential overlap is obtained by numerically integrating the slip velocity of the colliding surfaces.

Normal damping coefficient $\eta_{n}$ is determined according to the coefficient of restitution (COR) so that the actual COR is independent of the initial relative velocity of the particles (Antypov \& Elliott 2011). Without better knowledge, the tangential damping coefficient is set equal to the normal damping coefficient. The equations given in table 1 are used to model both particle-particle and particle-wall contacts. For particle $i$ in contact with a planar wall, the curvature radius and the mass of the wall are large when compared to the particle, so effectively $r^{*}=r_{i}$ and $m^{*}=m_{i}$. 
Normal forces

Contact force

Relative velocity

$$
\begin{gathered}
\boldsymbol{f}_{c, i j}^{n}=k_{n} \delta_{n}^{3 / 2} \boldsymbol{n}_{i j}-\eta_{n} \boldsymbol{v}_{i j}^{n} \\
\boldsymbol{v}_{i j}^{n}=\left(\left(\boldsymbol{v}_{i}-\boldsymbol{v}_{j}\right) \cdot \boldsymbol{n}_{i j}\right) \boldsymbol{n}_{i j} \\
k_{n}=\frac{4}{3} Y^{*} \sqrt{r^{*}}
\end{gathered}
$$

Stiffness coefficient

Damping coefficient

$$
\eta_{n}=-\ln (\mathrm{COR}) \sqrt{\frac{5}{\ln (\mathrm{COR})^{2}+\pi^{2}}}\left(m^{*} k_{n}\right)^{1 / 2} \delta_{n}^{1 / 4}
$$

Tangential forces

Contact force

$$
\boldsymbol{f}_{c, i j}^{t}= \begin{cases}-k_{t} \boldsymbol{\delta}_{t}-\eta_{t} \boldsymbol{v}_{i j}^{t}, & \text { if } \boldsymbol{f}_{c, i j}^{t}<\mu_{s}\left|\boldsymbol{f}_{c, i j}^{n}\right| \\ -\mu_{s}\left|\boldsymbol{f}_{c, i j}^{n}\right| \frac{\boldsymbol{v}_{i j}^{t}}{\left|\boldsymbol{v}_{i j}^{t}\right|}, & \text { if } \boldsymbol{f}_{c, i j}^{t} \geqslant \mu_{s}\left|\boldsymbol{f}_{c, i j}^{n}\right|\end{cases}
$$

Slip velocity

$$
\boldsymbol{v}_{i j}^{t}=\boldsymbol{v}_{i j}-\boldsymbol{v}_{i j}^{n}+\left(r_{i} \boldsymbol{\omega}_{i}+r_{j} \boldsymbol{\omega}_{j}\right) \times \boldsymbol{n}_{i j}
$$

Stiffness coefficient

Damping coefficient

$$
k_{t}=8 G^{*} \sqrt{r^{*} \delta_{n}}
$$

$$
\eta_{t}=\eta_{n}
$$

Equivalent properties

$$
(v=\text { Poisson's ratio })
$$

Elastic modulus

$$
Y^{*} \equiv\left(\frac{1-v_{i}^{2}}{Y_{i}}+\frac{1-v_{j}^{2}}{Y_{j}}\right)^{-1}
$$

Shear modulus

$$
G^{*} \equiv\left(\frac{1-v_{i}}{G_{i}}+\frac{1-v_{j}}{G_{j}}\right)^{-1}
$$

Mass

$$
\begin{aligned}
m^{*} & \equiv\left(\frac{1}{m_{i}}+\frac{1}{m_{j}}\right)^{-1} \\
r^{*} & \equiv\left(\frac{1}{r_{i}}+\frac{1}{r_{j}}\right)^{-1}
\end{aligned}
$$

TABLE 1. The contact forces and the equivalent properties for particles $i$ and $j$ in contact.

The motion of the gas phase is solved in terms of the locally averaged variables over the computational mesh by solving the continuity and momentum equations of an incompressible fluid:

$$
\begin{gathered}
\frac{\partial \alpha}{\partial t}+\nabla \cdot \alpha \boldsymbol{u}=0 \\
\rho \frac{\partial(\alpha \boldsymbol{u})}{\partial t}+\rho \nabla \cdot(\alpha \boldsymbol{u} \boldsymbol{u})=\rho \boldsymbol{\nabla} \cdot \alpha \boldsymbol{\tau}-\nabla p+\rho \alpha \boldsymbol{g}-\boldsymbol{F}_{g \rightarrow p},
\end{gathered}
$$

where $\alpha, p$ and $\rho$ are the volume fraction, pressure and mass density of the gas phase, respectively; $\boldsymbol{u}$ is the velocity of the gas, $\boldsymbol{\tau}$ is the gas phase deviatoric stress tensor and $\boldsymbol{F}_{g \rightarrow p}$ is the local gas-particle interaction force per unit volume.

The forces exerted by the gas on the particles are calculated on a per-particle basis and an opposite force is applied to the gas. The force per unit volume exerted by the gas on the particles in a cell is given by

$$
\boldsymbol{F}_{g \rightarrow p}=\frac{1}{\Delta V} \sum_{i} \boldsymbol{f}_{g \rightarrow p, i},
$$


where $\Delta V$ is the volume of the cell and the sum runs over the particles in the cell. Neglecting the local acceleration of the fluid $(\mathrm{D} \boldsymbol{u} / \mathrm{D} t=0)$, the total force exerted on an individual particle can be written as

$$
\boldsymbol{f}_{g \rightarrow p, i}=\left(\boldsymbol{f}_{d, i}+\boldsymbol{f}_{g \rightarrow p, i}^{\prime}\right) / \alpha-\rho V_{p, i} \boldsymbol{g},
$$

where $\boldsymbol{f}_{d, i}$ is the drag force and $\boldsymbol{f}_{g \rightarrow p, i}^{\prime}$ includes all the remaining gas-particle interaction forces such as the virtual mass effect and the Basset force (Zhou et al. 2010).

Due to their low importance in gas-solid flows, the forces included in $\boldsymbol{f}_{g \rightarrow p, i}^{\prime}$ are neglected in this study. The Wen \& Yu (1966) drag model is used to account for the effect of the local particle volume fraction on the drag force:

$$
\boldsymbol{f}_{d, i}=\frac{3}{4} \frac{\rho V_{i}}{d_{i}} C_{d}\left(\boldsymbol{u}_{i}-\boldsymbol{v}_{i}\right) \alpha^{-1.65},
$$

where $V_{i}$ and $d_{i}$ are the volume and the diameter of the particle, $C_{d}$ is the drag coefficient, $\boldsymbol{u}_{i}$ is the flow velocity interpolated at the particle position and $\boldsymbol{v}_{i}$ is the particle velocity. The drag coefficient is evaluated based on the Schiller \& Naumann (1933) correlation as formulated by Rowe (1961):

$$
C_{d}= \begin{cases}\frac{24}{R e_{i}}\left(1+0.15 R e_{i}^{0.687}\right) & \text { if } R e_{i}<1000 \\ 0.44 R e_{i} & \text { if } R e_{i} \geqslant 1000\end{cases}
$$

where

$$
\operatorname{Re}_{i}=\frac{\alpha \rho\left|\boldsymbol{u}_{i}-\boldsymbol{v}_{i}\right| d_{p, i}}{\mu}
$$

is the particle Reynolds number.

\subsection{Triboelectric charging}

The term 'effective work function difference' stems from the well-established theory of contact charging between metals, where the total charge transferred between two objects is proportional to the work function difference of the surfaces (i.e. the difference in the energies required to extract an electron from each surface). In the case of ideal insulators, electrons are localized and participate in chemical bonding between molecules. In terms of band theory, it is very unlikely that an electron will transfer from a valence state on one surface to a conduction state on another, as the energy gap between the valence and conduction bands is wide. However, the band theory is theoretically valid only for continuous, homogeneous systems, so contact charging of insulators has been associated with electron states present at the surfaces only (Lowell \& Rose-Innes 1980; Castle 1997; Mehrani, Bi \& Grace 2007; Lacks \& Sankaran 2011; Fotovat et al. 2017b).

The surface state theory (Lowell \& Rose-Innes 1980) describes the charge transfer between two insulating surfaces based on the amount of available donor and acceptor states on the surfaces. Originally the theory was proposed for electron transfer, but it applies conceptually to ion transfer as well (Lee 1994). The numerical model proposed by Laurentie et al. $(2010,2013)$ is based on so-called high-density limit of the surface state theory: it is assumed that the net charge exchange between surfaces is not limited by the density of the available donor and acceptor states but rather by the electric field developed in the contact gap when the surfaces are separated. 


\subsubsection{Numerical model}

Based on Laurentie et al. (2013), the charge exchange between two objects (either two particles or a particle and a wall) due to a discrete change in the contact area is given by

$$
\frac{\Delta q_{i j}}{\Delta A_{i j}}=\varepsilon\left(\frac{\Delta \varphi_{j i}}{\delta_{c} e^{+}}+\boldsymbol{E}_{i j} \cdot \boldsymbol{n}_{i j}\right),
$$

where $\Delta q_{i j}$ is the charge transferred to object $i$ from object $j, \Delta A_{i j}$ is the change in the contact area, $\varepsilon$ is the permittivity of the gas, $\Delta \varphi_{j i}=\varphi_{j}-\varphi_{i}$ is the effective work function difference between the surfaces, $\delta_{c}$ is the critical distance over which charge can transfer, $e^{+}$is the elementary (positive) charge and $\boldsymbol{E}_{i j}$ is the electric field at the contact point without the contribution of the charge transferred during the contact. In line with Laurentie et al. $(2010,2013)$, charge transfer is restricted to the period during which the contact area is increasing $\left(\Delta A_{i j}>0\right)$. This ensures that the total charge transferred during a collision is proportional to the maximum contact area.

The critical distance $\delta_{c}$ is an approximate distance over which charge transfer is reasonably probable. The values of $\delta_{c}$ found in the literature vary significantly and the value generally depends on environmental conditions such as humidity and, of course, the actual species responsible for the charge transfer (McCarty \& Whitesides 2008). As the total charge transferred during a contact depends only on the ratio $\Delta \varphi /\left(\delta_{c} e^{+}\right)$, it was not considered reasonable to choose an explicit value for $\delta_{c}$ in this study. Instead, $\Delta \varphi /\left(\delta_{c} e^{+}\right)$is used as a lumped parameter in the simulations, including the influence of both the effective work function difference and the critical distance. According to (2.10), this parameter can be interpreted as the electric field strength required to suppress charge transfer.

The advantage of this triboelectric charging model is its compatibility with the discrete element model. The change in contact area of the colliding objects is approximated from the change in the normal overlap distance $\delta_{n, i j}$ as (Kolehmainen et al. 2017a)

$$
\Delta A_{i j}=2 \pi r^{*} \Delta \delta_{n, i j},
$$

which is straightforward to evaluate as the normal overlap distance is already required by the contact model. Note that possible change in contact area due to the relative tangential velocity of the colliding surfaces is not taken into account in (2.11).

To speed up the simulations, a relatively low Young's modulus is applied to the particles so as to increase the average collision time. This increases the average contact area and hence the average charge transferred in each collision. Based on the soft-sphere contact model, Kolehmainen et al. (2017a) concluded that if damping forces are neglected, the ratio of the charge that would be transferred in a real contact to the charge transferred in a simulated 'soft' contact is

$$
a_{\text {corr }}=\frac{\Delta q_{\text {real }}}{\Delta q_{\text {soft }}}=\left(\frac{Y_{\text {soft }}^{*}}{Y_{\text {real }}^{*}}\right)^{2 / 5} .
$$

As long as the particle softening does not alter significantly the dynamics of the bed, the effect of particle softening can be eliminated by multiplying the charge transfer rate given by (2.10) with $a_{\text {corr }}$ (Kolehmainen et al. 2017a).

To achieve feasible simulation times, the charge transfer rate given by (2.12) is further scaled by an artificial acceleration factor $a$. In earlier studies by Kolehmainen et al. $(2017 a, b)$, the acceleration factor did not affect the saturation charge significantly. However, artificially increasing the charge transfer rate distorts the charge distribution, increasing the proportion of charge on the most strongly charged particles. This is further discussed in later sections. 


\subsection{Equilibrium charge}

In this study, the equilibrium charge is defined as the maximum charge that a particle can acquire by means of triboelectric charging when in contact with an uncharged and unpolarized insulating wall. In terms of the present charging model, equilibrium is achieved when the charge transfer is suppressed by the electric field induced by the particle itself.

Based on (2.10) no charge is transferred during a particle-wall contact when

$$
\frac{\Delta \varphi_{w p}}{\delta_{c} e^{+}}+E_{\perp, w p}=0,
$$

where $\Delta \varphi_{w p}=\varphi_{w}-\varphi_{p}$ is the effective work function difference between the wall and the particle and $E_{\perp, w p}$ is the electric field strength normal to the wall at the contact point (pointing towards the particle). Neglecting any external electric field and assuming that the particle is perfectly spherical and uniformly charged, the electric field due to the particle itself is $E_{\perp, w p}=-q_{e q} /\left(\pi \varepsilon d_{p}^{2}\right)$, where $q_{e q}$ is the equilibrium charge. Substituting to (2.13) and rearranging gives the equilibrium charge

$$
q_{e q}=\pi \varepsilon d_{p}^{2}\left(\frac{\Delta \varphi_{w p}}{\delta_{c} e^{+}}\right) .
$$

When multiple particles are involved, the level at which the mean particle charge saturates is limited by the total electric field developed in the bed. Also the electric field due to a charged wall further restricts accumulation of charge on particles; consequently, the mean particle charge in a bed can be considerably smaller than $q_{e q}$.

In accordance with Kolehmainen et al. $(2017 a, b)$, the expected magnitude of electrostatic effects is characterized by the dimensionless quantity $e / g$ (here $e / g$ is a symbol and does not equal to $e^{+} / g$ ), which is the ratio of the magnitude of the electrostatic force between two particles with equilibrium charge to the gravitational force:

$$
e / g \equiv \frac{q_{e q}^{2} /\left(4 \pi \varepsilon d_{p}^{2}\right)}{m g},
$$

where $m$ is the mass of a particle. Together with (2.14), this parameter can be used to estimate the importance of electrostatic forces in the bed with a given effective work function difference.

\subsection{Electrostatic forces}

Assuming that the charge on particle $i$ is uniformly distributed on its surface and neglecting possible non-uniformity of the electric field over the particle, the electrostatic force acting on the particle is given by

$$
\boldsymbol{f}_{e, i}=q_{i} \boldsymbol{E}_{i}
$$

where $q_{i}$ is the charge of the particle and $\boldsymbol{E}_{i}$ is the electric field evaluated at the centre of the particle. When charge on the particle surfaces is uniformly distributed, the particles can be treated as point charges when evaluating the electric field (Feynman, Leighton \& Sands 1966): for example, the electric field on particle $i$ due to particle $j$ is given by

$$
\boldsymbol{E}_{j \rightarrow i}=\frac{q_{j}}{4 \pi \varepsilon} \frac{\boldsymbol{x}_{i}-\boldsymbol{x}_{j}}{\left|\boldsymbol{x}_{i}-\boldsymbol{x}_{j}\right|^{3}},
$$

where $q_{j}$ is the charge of particle $j$ and $\boldsymbol{x}$ denotes the position of the particle indicated by the subscript. 
The electric field affecting on each particle is evaluated based on a particle-particle particle-mesh (PPPM) method, which in general is based on mapping particles to a computational mesh and solving a potential over the mesh (according to a relevant discretized Poisson equation). Long-range interactions between particles are then solved by interpolating the field from the mesh to each particle, but interactions between particles close to each other are evaluated through a direct sum (Toukmaji $\&$ Board 1996).

In accordance with Kolehmainen, Ozel \& Sundaresan (2016), Kolehmainen et al. $(2017 a, b)$, the electric field affecting particle $i$ is expressed as a sum a short-range term $\boldsymbol{E}_{s, i}$, a long-range term $\boldsymbol{E}_{\nabla^{2}, i}$ and a correction term $\boldsymbol{E}_{c, i}$, which is used to remove the overlapping contribution of the former two. The short-range term is evaluated as a direct contribution due to the nearby particles as

$$
\boldsymbol{E}_{s, i}=\frac{1}{4 \pi \varepsilon} \sum_{j} q_{j} \frac{\boldsymbol{x}_{i}-\boldsymbol{x}_{j}}{\left|\boldsymbol{x}_{i}-\boldsymbol{x}_{j}\right|^{3}} .
$$

In this study, the procedure for solving the short-range electric field is adapted to the DEM implementation of OpenFOAM. A list of so-called interacting cells is constructed at the beginning of the simulations to track the location of the particles in the computational mesh. The short-range term is not limited by a prescribed cutoff radius as in Kolehmainen et al. $(2016,2017 a, b)$ but covers all the particles located in the interacting cells. The list of interacting cells is determined according to the desired minimum distance of the short-range term $\left(\delta_{s, m i n}\right)$. For example, all the particles in cell $A$ contribute to the short range field of the particles in cell $B$ if the shortest distance between the cells is below $\delta_{s, m i n}$.

Similarly to Kolehmainen et al. (2016, 2017a,b), the long-range field is evaluated as the gradient of the electric potential $\phi$ over the computational mesh:

$$
\boldsymbol{E}_{\nabla^{2}}=-\nabla \phi .
$$

The electric potential, in turn, is solved from a Poisson equation

$$
\nabla^{2} \phi=-\frac{\rho_{c}}{\varepsilon},
$$

where $\rho_{c}$ is the volumetric charge density based on the total charge in each cell and $\varepsilon$ is the mixture permittivity.

A correction term is used to remove the contribution of the charge already involved in the short-range term from the long-range term. This is done by approximating the contribution of the charge in the interacting cells to the long-range field in each cell. The correction term for particles in cell $A$ is

$$
\boldsymbol{E}_{c, A}=-\frac{1}{4 \pi \varepsilon} \sum_{B} Q_{B} \frac{\boldsymbol{x}_{A}-\boldsymbol{x}_{B}}{\left|\boldsymbol{x}_{A}-\boldsymbol{x}_{B}\right|^{3}},
$$

where the sum is taken over all interacting cells of cell $A, Q_{B}$ is the total charge in interacting cell $B$ and $\boldsymbol{x}$ indicates the location of the cell centre indicated by the subscript. Differing from Kolehmainen et al. (2016, 2017a,b), the correction term is equal for all particles in a cell, so the electric field correction can be conducted cell by cell before mapping the long-range field to the particles.

In order to evaluate the charge transfer between particles, the electric field at the contact point of particles $i$ and $j$ is approximated as

$$
\boldsymbol{E}_{i j} \cdot \boldsymbol{n}_{i j}=\boldsymbol{E}_{r, i j} \cdot \boldsymbol{n}_{i j}-q_{i} /\left(\pi \varepsilon d_{p, i}^{2}\right)+q_{j} /\left(\pi \varepsilon d_{p, j}^{2}\right),
$$


where $\boldsymbol{E}_{r, i j}$ is the electric field due to other sources than the colliding particles. $\boldsymbol{E}_{r, i j}$ is linearly interpolated from electric fields at the particle centres as

$$
\boldsymbol{E}_{r, i j}=\frac{\left(\boldsymbol{E}_{i}-\boldsymbol{E}_{j \rightarrow i}\right) d_{i}+\left(\boldsymbol{E}_{j}-\boldsymbol{E}_{i \rightarrow j}\right) d_{j}}{d_{i}+d_{j}} .
$$

In the case of a particle-wall contact, the electric field strength at the contact point is approximated as $\boldsymbol{E}_{i w} \cdot \boldsymbol{n}_{i w}=\boldsymbol{E}_{i} \cdot \boldsymbol{n}_{i w}-q_{i} /\left(\pi \varepsilon d_{p, i}^{2}\right)$, where $\boldsymbol{E}_{i}$ is the electric field evaluated at the centre of the particle and $\boldsymbol{n}_{i w}$ is an unit normal vector pointing from the contact point towards the particle.

\subsection{Charge on the wall}

Whenever a particle exchanges charge with an insulating wall, an opposite charge remains on the contact area. This in turn affects the electric field developed in the bed. In this study, the wall charge is accounted in the electric field solved over the computational mesh. In principle, the electric potential is solved from (2.20) on both sides of the wall with using an appropriate boundary condition at the interface.

In homogeneous, linear and isotropic dielectric media the polarization is aligned and proportional to the electric field. At the interface of dielectric media $A$ and $B$ holds

$$
\boldsymbol{n}_{A B} \cdot\left(\varepsilon_{B} \boldsymbol{E}_{B}-\varepsilon_{A} \boldsymbol{E}_{A}\right)=\sigma,
$$

where $\boldsymbol{n}_{A B}$ is an unit normal vector of the interface pointing from $A$ to $B ; \varepsilon_{A}, \varepsilon_{B}$, $\boldsymbol{E}_{A}$ and $\boldsymbol{E}_{B}$ are the permittivities and the electric fields in mediums $A$ and $B$ in the immediate vicinity to the point considered and $\sigma$ is the free charge density on the surface (Jackson 1999).

The charge on each boundary face of the computational mesh is tracked assuming an uniform charge distribution over the face. The electric potential of a boundary face between adjacent cells $A$ and $B$ is obtained by discretizing (2.24), resulting in

$$
\phi_{f}\left(\frac{\varepsilon_{B}}{d_{B}}+\frac{\varepsilon_{A}}{d_{A}}\right)=\varepsilon_{B} \frac{\phi_{B}}{d_{B}}+\varepsilon_{A} \frac{\phi_{A}}{d_{A}}+\sigma_{f},
$$

where $\phi_{f}$ is the potential at the boundary face, $\phi_{A}$ and $\phi_{B}$ are the potentials at the respective cell centres, $d_{A}$ and $d_{B}$ are the perpendicular distances from the face to the respective cell centres and $\sigma_{f}$ is the free surface charge density on the face (see figure 1). In (2.25) the normal component of the electric field on both sides of the face has been linearly approximated from the potentials at the face and at the adjacent cell centre.

\section{Experimental set-up}

Spherical polyethylene particles (Cospheric, CPMS-0.96) were fluidized by nitrogen gas in a rectangular soda-lime glass column. Based on the number frequency distribution provided by the manufacturer, the particle size distribution obeys approximately a truncated normal distribution described in table 2 .

The main components of the experimental set-up are schematically depicted in figure 2. The fluidizing device consists of a rectangular fluidization column (A) and a plastic base part (B). The gas flows through a removable plastic inlet (C) and leaves through a conical expansion (D). The dimensions of the fluidization column are given in table 2. 


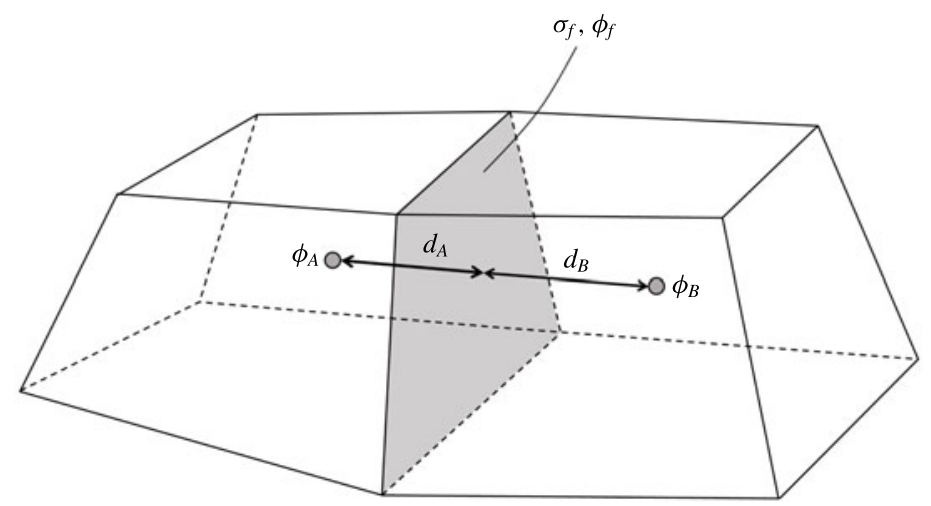

Figure 1. Common face of cells $A$ and $B$ at a region interface.

Particle properties

Size distribution:

Diameter range

Mean value

Mode

Standard deviation

Density

$\begin{array}{cc}240-305 & \mu \mathrm{m} \\ 276 & \mu \mathrm{m} \\ 277 & \mu \mathrm{m} \\ 13.7 & \mu \mathrm{m} \\ 960 & \mathrm{~kg} \mathrm{~m}^{-3}\end{array}$

Device dimensions

Column cross-section

Inlet cross-section

Column height

$\begin{array}{cc}10 \times 10 & \mathrm{~mm}^{2} \\ 6 \times 6 & \mathrm{~mm}^{2} \\ 740 & \mathrm{~mm}\end{array}$

PIV set-up

Imaged area (distance from inlet)

Illumination angle

Cycle interval

$\pi / 4$

1000

$\mathrm{mm}$

Pulses per cycle

5

Pulse length

$2 \mu s$

Pulse interval

Image resolution

Interrogation window size

$\begin{array}{rc}2 & \mu s \\ 5 & \mu s \\ 2000 \times 2000 & \text { pixels } \\ 128 \times 128 & \text { pixels }\end{array}$

TABLE 2. Particle properties, dimensions of the fluidizing device and parameters used for particle image velocimetry.

The inlet is a square duct covered by a stainless steel grating. Both the glass pipe and the inlet part are removable, which enables collecting the particles after fluidization. The total charge of the particles was measured before and after fluidization using a Faraday cup connected to an electrometer (Monroe 284).

The gas flow rate is controlled by a mass flow controller (Bronkhorst, F201CV). A part of the gas is conveyed through a humidifier $(\mathrm{E})$, while the other part is conveyed directly into the base part (B). The portion of the gas flowing through the humidifier is manually controlled using a rotameter $(F)$ and a ball valve $(\mathrm{G})$. The relative humidity is measured from the base part using a relative humidity and temperature transmitter (Omega HX94 SS RH Probe). 


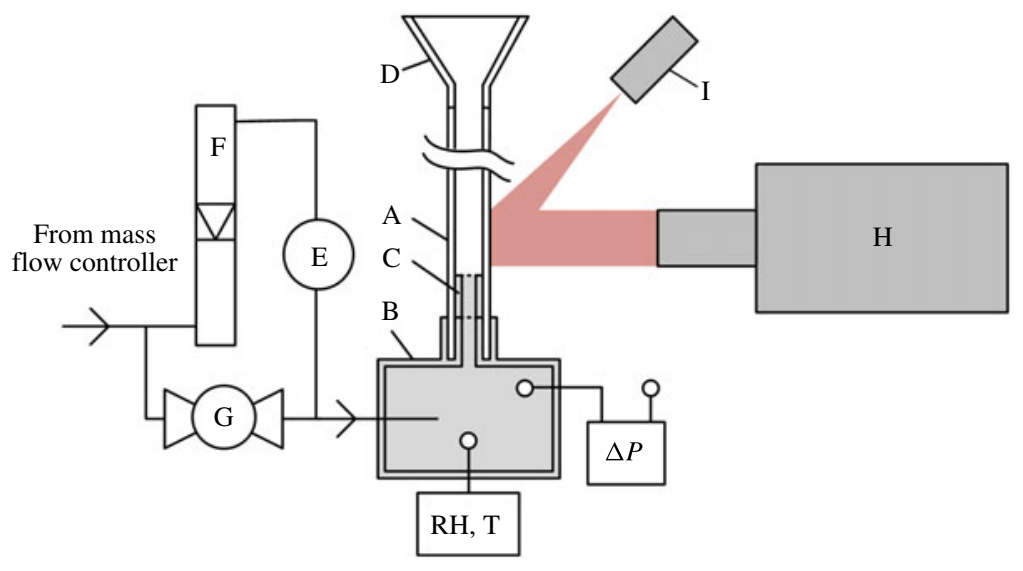

FIgURE 2. (Colour online) The fluidizing device and the instruments used. A: glass pipe, B: base part, C: removable inlet, D: conical expansion, E: humidifier, F: rotameter, G: ball valve, H: high-speed camera, I: laser.

The pressure difference between the base part and the ambient air is measured by a differential pressure sensor (Sensirion SDP1108). The hydrostatic pressure difference due to the density difference between nitrogen and the ambient air is insignificant (under $0.5 \mathrm{~Pa}$ ), so the measured pressure difference is virtually equal to the overall friction loss across the fluidization device. The pressure loss measured across the empty device is subtracted from the pressure loss measured in each experiment to determine the loss due to the fluidized bed only.

The wall layer was imaged with a high-speed camera (pco dimax HS4) in selected humidities; the humidities were chosen so that the behaviour of the bed is expected to differ between the imaged cases. The bed was illuminated with a pulsed diode HF laser (Cavitar Cavilux HF), giving a cycle of short pulses between time intervals. PIVlab (Thielicke \& Stamhuis 2014) was used to estimate the velocity of the particles in the imaged area in terms of particle image velocimetry (PIV). The imaged area covers the range of $15-30 \mathrm{~mm}$ above the bed inlet and the interrogation window size corresponds to a physical size of about three particles. Other essential parameters of the PIV set-up are given in table 2 .

A set of experiments was carried out in relative humidities ranging from 0 to $60 \%$. Besides the final charge and mass of the particles, the pressure drop across the bed was measured. The duration of each experiment was $30 \mathrm{~min}$, which was considered sufficient for the charge to saturate in most conditions. The relative humidity was kept in the range of $\pm 1 \%$ of the desired value during each experiment.

The flow rate of the fluidizing gas was $5.4 \times 10^{-5} \mathrm{~m}^{3} \mathrm{~s}^{-1}$ in each experiment, corresponding to a superficial velocity of approximately $50 \%$ of the settling velocity of an average-sized particle. The mass of the particles was measured before each experiment and after measuring the final charge, being $1.5 \pm 0.03 \mathrm{~g}$. The final mass was used to determine the final charge-to-mass ratio of the particles.

The same set of particles was used in successive experiments, but new particles were added to cover the minor loss of particles during the experiments. The charge on the particles was erased between the experiments by briefly fluidizing them in a high relative humidity ( $>60 \%$ ), so that the charge before the next experiment was between $\pm 3 \mathrm{nC} \mathrm{g}^{-1}$. To reasonably estimate the effect of the charge accumulated on 


\begin{tabular}{lcc} 
& \multicolumn{2}{c}{ Contact } \\
\cline { 2 - 3 } & Particle-particle & Particle-wall \\
$Y^{*}$ & $0.063 \mathrm{MPa}$ & $0.057 \mathrm{MPa}$ \\
$\mu_{s}$ & 0.5 & 0.5 \\
COR & 0.9 & 0.9 \\
$a_{\text {corr }}$ & 0.033 & 0.024
\end{tabular}

TABLE 3. The characteristic properties for different contact types.

the particles to the pressure drop across the bed, the average pressure drop over the last $20 \mathrm{~s}$ of each experiment were compared to the time-averaged pressure drop across an effectively uncharged bed (where the final absolute net charge was measured to be smaller than $1 \mathrm{nC} \mathrm{g}^{-1}$ ).

\section{Simulation set-up}

\subsection{Geometry and boundary conditions}

The computational mesh covers the fluidization column from the inlet to the conical expansion and consists of a grid of $1 \mathrm{~mm}^{3}$ cubical cells. The dimensions of each cell correspond to approximately three particle diameters, which typically provides nearly grid-independent results in terms of particle-fluid interaction (Radl \& Sundaresan 2014) and the PPPM method used to evaluate the electrostatic forces (Kolehmainen et al. 2016). The minimum range of the short-range electric field was set slightly smaller than $1 \mathrm{~mm}$, so only the particles in immediately neighbouring cells contribute to the short-range electric field experienced by each other.

For accounting the effect of surface charge on the walls the simulated bed was enclosed in a cylindrical outer region with a diameter of $200 \mathrm{~mm}$. The electric potential was fixed at zero at the outer boundaries of the exterior region and (2.25) was applied at the interface of the regions. Vacuum permittivity was assumed in both regions, thus ignoring the effect of polarization of the wall and the particles. The effect of the metal grating at the bottom of the bed was not taken into account but was treated as a glass surface. The total charge accumulated to the metal grating at the bottom of the bed was found relatively small both in the simulations $(<0.15 \mathrm{nC})$ and in the experiments $(<2.5 \mathrm{nC})$.

A uniform flow velocity boundary condition was invoked at the flow inlet and a constant pressure boundary condition at the outlet. The density and viscosity of the fluid were set equal with those of dry nitrogen at $297.15 \mathrm{~K}$. The initial state of the simulations was full fluidization of $140400(\approx 1.5 \mathrm{~g})$ initially uncharged particles.

\subsection{Particle properties}

Particle size distribution used in the simulations obeys a truncated normal distribution described in table 2. To achieve reasonable simulation times, a low Young's modulus $(0.1 \mathrm{MPa})$ is applied on both the particles and the wall while the Poisson's ratio is 0.46 for the particles and 0.22 for the wall. Other characteristics of particle-particle and particle-wall contacts are given in table 3 .

The values of Young's moduli found in the literature range from 0.5 to $1.2 \mathrm{GPa}$ for polyethylene and from 50 to $90 \mathrm{GPa}$ for glass. This affects the charge transfer rate so that the charge transfer correction factor $a_{\text {corr }}$ calculated from (2.12) is in the range 


$\begin{array}{lcccc}e / g & \frac{\Delta \varphi_{w p}}{\delta_{c} e^{+}}\left(\mathrm{V} \mathrm{nm}^{-1}\right) & \Delta \varphi_{w p}(\mathrm{eV})^{a} & \Delta \varphi_{w p}(\mathrm{eV})^{b} & Q_{\infty} / M\left(\mathrm{nC} \mathrm{g}^{-1}\right) \\ 0 & 0 & 0 & 0 & \\ 2 & 6.27 \times 10^{-4} & 6.27 \times 10^{-4} & 0.313 & 8.15 \\ 4 & 8.86 \times 10^{-4} & 8.86 \times 10^{-4} & 0.443 & 12.2 \\ 8 & 1.250 \times 10^{-3} & 1.25 \times 10^{-4} & 0.625 & 19.9\end{array}$

TABLE 4. The values of $e / g$ used in the preliminary simulations and corresponding effective work function differences with two different values of $\delta_{c}$ (basing on the mean particle diameter $\bar{d}_{p}$ ). The resulting charge-to-mass ratios are given in the last column.

$$
\begin{aligned}
& { }^{a} \delta_{c}=1 \mathrm{~nm} \text { (Lowell \& Rose-Innes 1980). } \\
& { }^{b} \delta_{c}=500 \mathrm{~nm} \text { (Laurentie et al. 2013). }
\end{aligned}
$$

of $0.023-0.033$ for a particle-particle contact and in the range of $0.017-0.024$ for a particle-wall contact. The correction factors used in the simulations (table 3) were calculated according to approximate real Young's modulus $0.5 \mathrm{GPa}$ for the particles and $74 \mathrm{GPa}$ for the wall. This uncertainty when determining $a_{\text {corr }}$ is considered acceptable as it affects only the charge transfer rate but not the equilibrium charge of individual particles.

\subsection{Determining the effective work function difference}

As discussed before, both the effective work function difference $\Delta \varphi_{w p}$ and the charge transfer cutoff distance $\delta_{c}$ are not known and are dependent, for example, on the relative humidity. For this reason, the lumped parameter $\Delta \varphi_{w p} /\left(\delta_{c} e^{+}\right)$is considered instead of absolute values of $\Delta \varphi_{w p}$. For convenience, $\Delta \varphi_{w p} /\left(\delta_{c} e^{+}\right)$is termed the effective work function difference in the rest of the text.

To reasonably compare simulated and experimental results, the total charge in the simulated bed should saturate to a level similar to that in experiments. To achieve this, a few preliminary simulations were conducted by choosing reasonable values of $e / g$ and calculating the corresponding values of $\Delta \varphi_{w p} /\left(\delta_{c} e^{+}\right)$from (2.14) and (2.15). To allow comparison with studies using a predefined value for $\delta_{c}$, work function differences corresponding to $\delta_{c}=1 \mathrm{~nm}$ (Lowell \& Rose-Innes 1980) and $\delta_{c}=500 \mathrm{~nm}$ (Laurentie et al. 2013) are given in table 4 .

The work function differences corresponding to the average charge-to-mass ratios at humidity levels of $0-60 \% \mathrm{RH}$ were linearly interpolated based on the effective work function differences and the saturated charge-to-mass ratios of the preliminary simulations. The interpolated work function differences were used in the subsequent simulations to produce similar charge-to-mass ratios as in the experiments.

\subsection{Time step and acceleration factor}

The time step used in the simulations is adjusted during run time so that the Courant number of the continuous phase does not exceed 0.5 , while the maximum time step is limited to $5 \times 10^{-5} \mathrm{~s}$. Furthermore, each time step is divided into a number of sub-steps to evaluate the collision model.

Ten different simulations were conducted using acceleration factor $a=10$ (including the preliminary simulations). For comparison, two simulations were also run using acceleration factors 2 and 5 and effective work function differences corresponding to 
$e / g=4$ and $e / g=8$. In each case the simulation time was limited to $a t=30 \mathrm{~s}$, which allows the total charge in the bed to effectively saturate.

Although the acceleration factor does not affect the saturation charge significantly, a high charge transfer rate distorts the charge distribution among the particles and causes excessive charge segregation. In order to study the effect of charge segregation, simulations were also performed with fixed charges, assuming that all the particles carry the same charge. Specifically, after running simulations with dynamic tribocharging (with $a=10$ ) until the total charge level saturated, the charge of each particle was set to the average value and tribocharging was disabled. The CFD-DEM simulations were then continued with this uniform charge distribution among the particles while maintaining the charge distribution of the wall.

\subsection{Determining pressure drop}

The pressure drop across the simulated bed was obtained from the difference of the average temporal pressures at the inlet and the outlet. Similarly to the experiments, the pressure drop across an empty simulated bed was subtracted from the overall pressure drop.

The final pressure drop across the bed was determined from the time-averaged pressure drop over the last $0.5 \mathrm{~s}$ of each simulation and is compared to the time-averaged pressure drop across an uncharged simulated bed. Although the absolute value of the pressure drop depends, for example, on the chosen drag model, the relative decrease in pressure drop due to particle-wall adhesion was found to be comparable with the experiments.

\section{Experimental results}

In favourable conditions, one can follow with the bare eye how a stationary layer of particles builds up along the walls. Typically, multiple layers of particles adhere on top of each other (figure 3).

It was found that particles acquire a relatively strong positive net charge in dry conditions but tend to charge negatively when relative humidity exceeds $30 \%$ (figure 4). The charge accumulated on the particles is related to the rate at which particles adhere to the wall: as shown in the inset of figure 4, the motion of particles on the wall ceases rapidly for $0 \%$ and $20 \%$ RH but shows not significant decrease at $30 \% \mathrm{RH}$. At $40 \% \mathrm{RH}$ the velocity of the wall layer approaches zero, although at a slower rate than in dry conditions.

As particles adhere to the wall, the pressure drop across the bed decreases. The relative decrease in pressure drop compared to the pressure drop across an uncharged bed is shown in figure 5. The inset shows a typical pressure drop in dry conditions, where the effects of tribocharging are the strongest.

\section{Simulation results}

The total charge of the simulated bed is found to obey the exponential saturation model (Liao, Hsiau \& Huang 2011; Pei, Wu \& Adams 2016; Kolehmainen et al. 2017a)

$$
Q(t) / M=Q_{\infty} / M\left(1-\mathrm{e}^{-a t / \tau_{Q}}\right),
$$

where $Q(t)$ is the total charge of the particles, $Q_{\infty}$ is the saturation charge, $a$ is the acceleration factor and $\tau_{Q}$ is a time scale depending on the intensity of particle-wall 
(a)

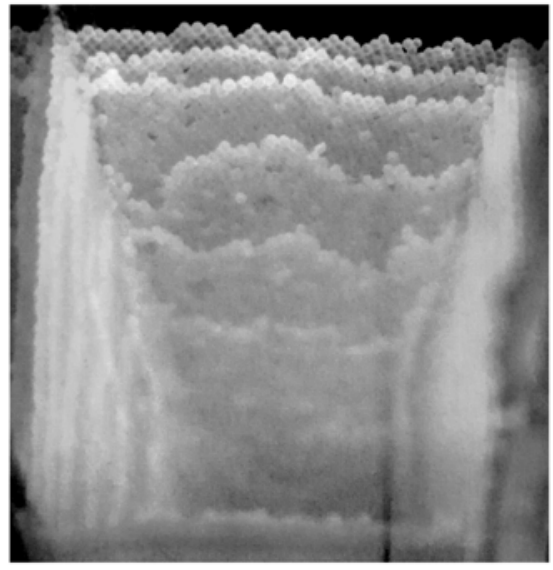

(b)

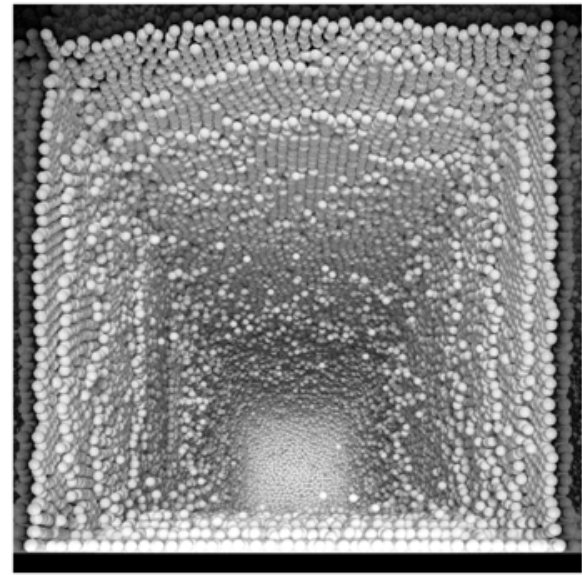

Figure 3. Particles adhered to the wall (viewed from the top of the bed). (a) Particles adhered after fluidization at $0 \%$ relative humidity. $(b)$ Adhered particles after simulation corresponding to $0 \% \mathrm{RH}$ with an uniform charge distribution among particles.

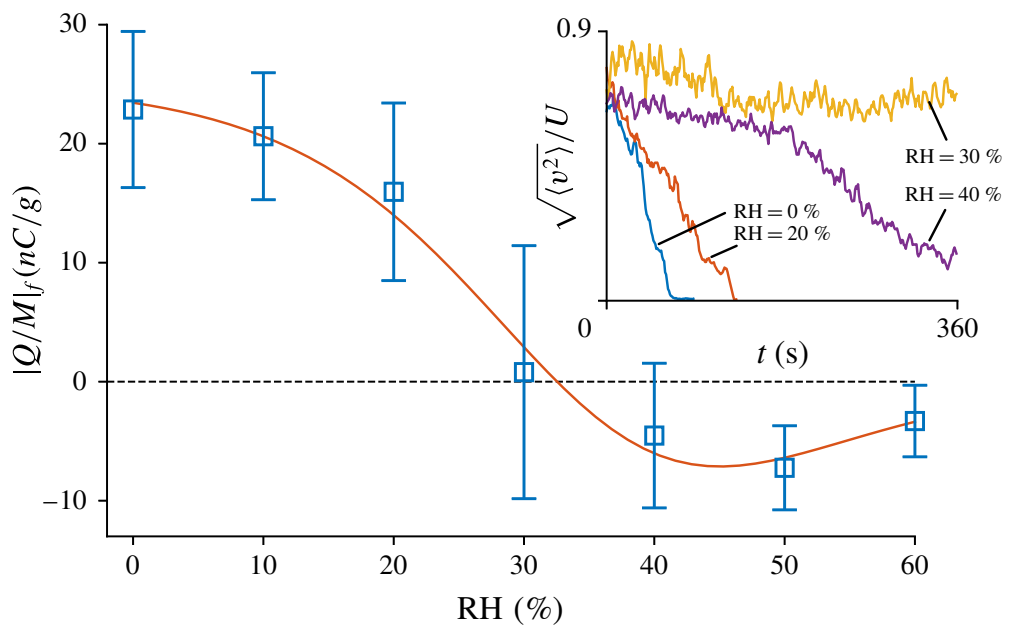

FIgURE 4. (Colour online) Final charge-to-mass ratio as a function of relative humidity. The solid curve is drawn to guide the eye and the error bars denote the $95 \%$ confidence intervals. Inset: the approximate root mean square speed of the particles at the wall in different relative humidities, scaled by the superficial velocity of the fluidizing gas.

contacts. The total charge is normalized by the total mass $M$ to allow comparison with the experimental charge-to-mass ratios.

The average saturation charge is insensitive to the acceleration factor as shown in figure 6 , but the charge distribution is visibly dependent on the acceleration factor. The charge on the majority of the particles is between 0 and $0.1 q_{e q}$, but there is a small peak between $0.3 q_{e q}$ and $0.5 q_{e q}$ due to the highly charged particles in the vicinity of the wall. The higher the acceleration factor, the further the peaks separate from each other; similar behaviour was also observed by Kolehmainen et al. (2017a). Both 


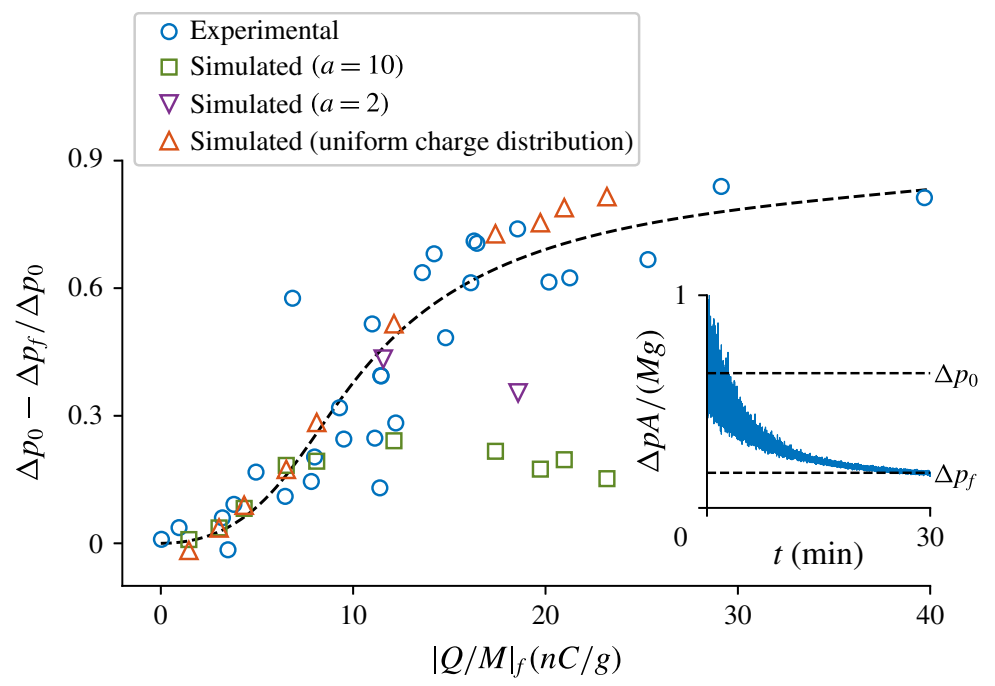

FIgURE 5. (Colour online) The relative decrease in the pressure drop across the bed as a function of the final absolute charge-to-mass ratio. $\Delta p_{0}$ is the average pressure drop over an uncharged bed and $\Delta p_{f}$ is the final pressure drop. The discrepancy between the experimental results and the simulation results with $a=10$ and $a=2$ is explained in 7 . Inset: pressure drop measured at $0 \% \mathrm{RH}$ (scaled by the total weight $M g$ of the particles and the cross-sectional area $A$ of the bed).

negatively and positively charged particles are present when $a \geqslant 5$, but in the case of $a=2$ the charge distribution is effectively unipolar as seen in figure 6 .

The charge-to-mass ratios obtained from the preliminary simulations are shown in figure 7 as a function of the effective work function difference. The solid black dots denote the work function differences interpolated (or extrapolated where necessary) according to the average experimental charge-to-mass ratios in different humidity levels.

The electrostatic forces in the wall-normal direction drive particles towards the wall. In the cases where $e / g \gtrsim 4$, a single layer of particles is rapidly drawn on the wall and the charge on the wall layer is segregated from the charge in the core region as depicted in figures 8 and 9. The wall-normal electrostatic force is strong enough to keep the particles on the wall when the fluidizing gas flow is stopped. In the case of fixed uniform charge distribution, multiple layers are left on the wall (figure 3). In contrast, when dynamic tribocharging is enabled, the first layer of particles carries greater-than-average charge (figure 9) and adheres more strongly, but multiple layer adhesion is not observed. Thus, a dynamic tribocharging model with an acceleration factor does not capture the formation of multiple particle layers next to the wall.

When a relatively high acceleration factor $(a=10)$ is used, the relative decrease in the pressure drop agrees with the experimental results up to approximately a $20 \%$ decrease of pressure drop (see figure 5). The particle number density near the wall rises when using a low acceleration factor $(a=2$, see figure 9), but still the decrease in the pressure drop does not adequately follow the experimental results with higher charge-to-mass ratios. The results obtained with $a=5$ are not shown as they are hardly different from the results obtained with $a=10$.

In the uniform charge case the average electrostatic force in the wall-normal direction experienced by the particles increases, especially near the wall (figure 9). 

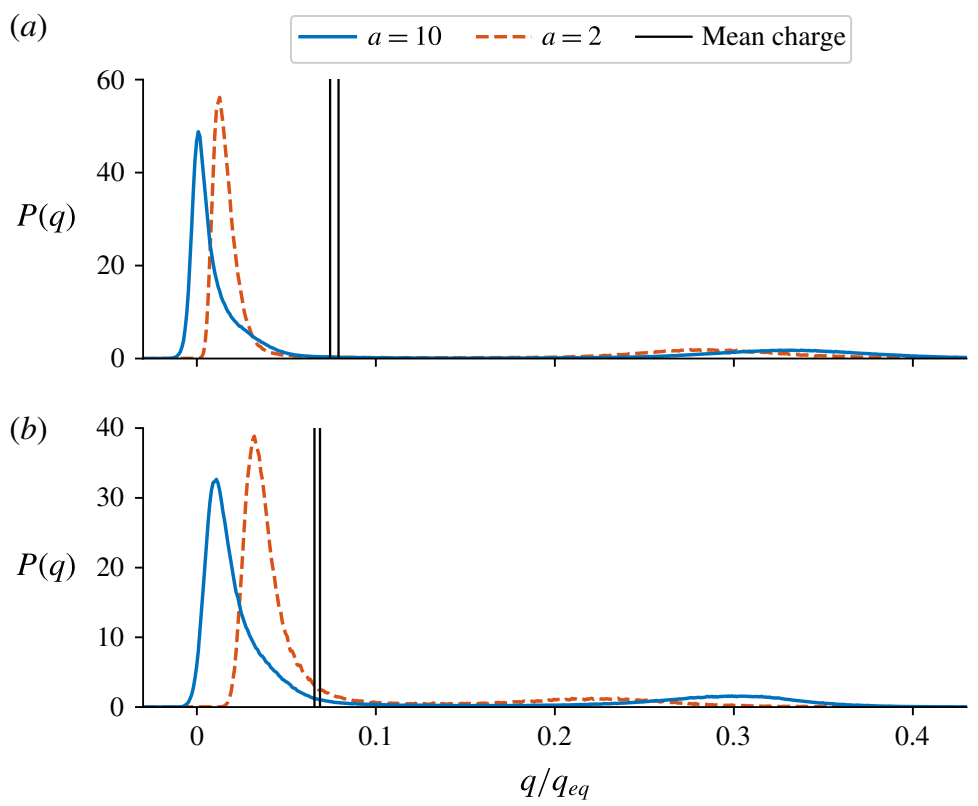

FIGURE 6. (Colour online) The probability density function of particle charge during the last $0.1 \mathrm{~s}$ of each simulation in cases of $e / g=8(a)$ and $e / g=4(b)$ with different acceleration factors. The solid black lines denote the minimum and maximum values of the mean particle charge among the cases $a=2, a=5$ and $a=10$.

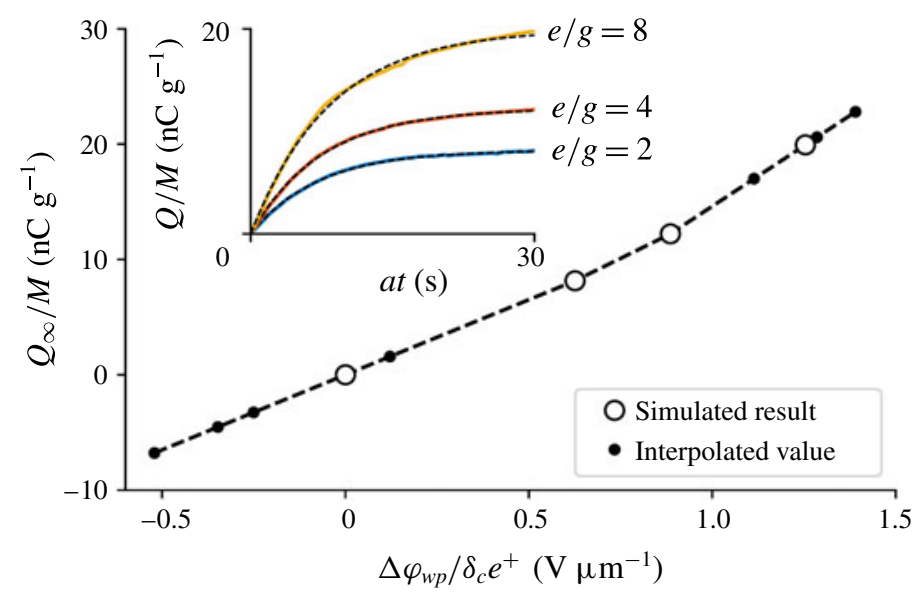

FIgURE 7. (Colour online) The saturation charges of the preliminary simulations and the interpolated work function differences. Inset: time evolution of the charge-to-mass ratios in the preliminary simulations.

As a result, particles experience appreciable attraction towards the wall over a large distance, resulting in a more realistic outcome in terms of accumulation of multiple particle layers on the wall and the associated decrease in pressure drop. The simulated pressure drop agrees remarkably well with the experimental results also with higher charge-to-mass ratios (figure 5). 
(a)

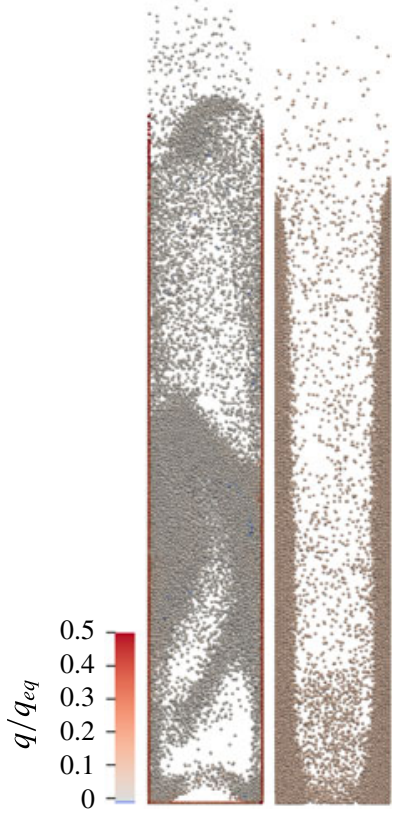

(b)

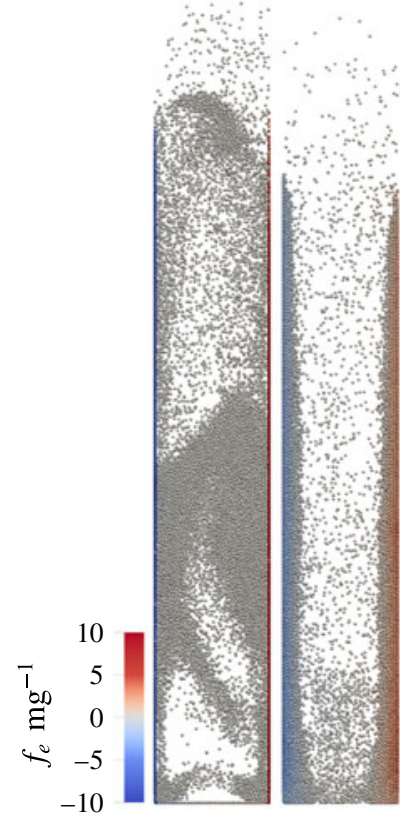

FIGURE 8. (Colour online) Snapshots from a simulation corresponding to $e / g=8$. (a) The ratio of particle charge to the equilibrium charge in the cases of dynamic tribocharging with $a=10$ (left) and fixed uniform charge distribution (right). (b) The ratio of the electrostatic force in vertical direction to the gravitational force in cases corresponding to $(a)$.

The root mean square speed (r.m.s. speed) of the particles on the wall is qualitatively similar to the experimental results. For example in simulations corresponding to $0 \%$ and $20 \% \mathrm{RH}$, the r.m.s. speed on the wall drifts towards zero, although at a significantly faster rate than in the experiments (compare figures 4 and 6). However, differing from the experimental results, the particle motion is not completely ceased but the r.m.s. speed level is approximately $0.04 \mathrm{~m} \mathrm{~s}^{-1}$. In corresponding uniform charge cases the r.m.s. velocity saturates at approximately 0.001 and $0.004 \mathrm{~m} \mathrm{~s}^{-1}$ in cases of $0 \%$ and $20 \% \mathrm{RH}$, respectively.

\section{Discussion}

The change of charge polarity in the experimental bed at approximately $30 \% \mathrm{RH}$ was unexpected, opposing our previous results obtained in vibrated bed experiments with similar particles (Kolehmainen et al. 2017b); this difference is probably due to different glass material (borosilicate) or different carrier gas (air) used in the earlier experiments. A humidity-dependent reversal of polarity has been earlier observed by Mehrani et al. (2007) for fines entrained from a fluidized bed of polyethylene particles. Although the origin of this change in polarity is not further considered in this text, it indicates a simultaneous action of more than one simultaneous charge transfer mechanisms.

As the pressure drop over the bed is proportional to the fluidized mass, figure 5 suggests that even $80 \%$ of particles may be supported by the wall or the base in such 

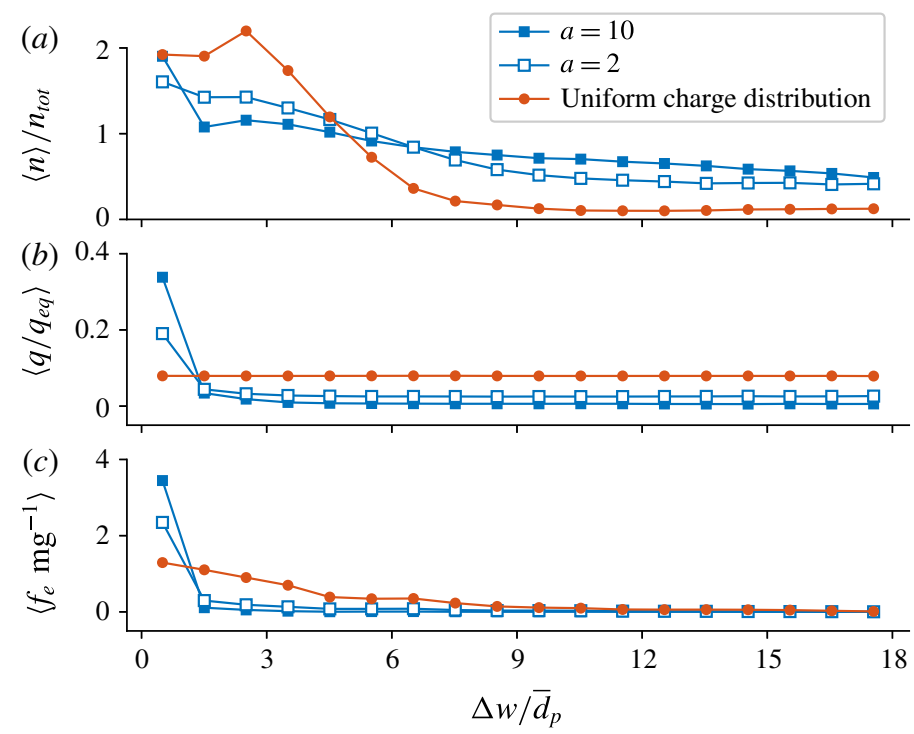

FIgURE 9. (Colour online) Time-averaged volumetric particle number density $n$, mean particle charge $q$ and the mean wall-normal electrostatic force $f_{e}$ in the case of $e / g=8$ (averaged over the last $0.1 \mathrm{~s}$ of each simulation and over the height of the fluidization column). $n_{\text {tot }}$ is the volumetric particle number density over the whole fluidization column, $\Delta w$ is the distance to the nearest vertical wall and $\bar{d}_{p}$ is the expected value of the particle diameter.

a narrow bed. The finding that in most of the simulations with dynamic tribocharging the relative decrease in the pressure drop is limited to approximately $20 \%$ is related to the fact that the amount of fluidized mass decreases only until the wall is covered by a single layer of particles.

The qualitative difference between the dynamic tribocharging and uniform charge cases is striking, as seen in figure 8. Excessive charge segregation in the tribocharging case restricts the development of multiple particle layers on the wall: basically, the attractive electrostatic field due to the wall is not strong enough to attach the more weakly charged particles onto the first layer. In addition, the strongly charged first layer exerts a significant repulsive field on the nearby particles. In contrast, the uniform charge case captures the continued decrease in the pressure drop with increasing charge level observed in the experiments. It is thus clear that our simulations with dynamic tribocharging overestimates the extent of charge segregation (i.e. the spread in charge probability density function).

The relatively slow development of the wall layer in the experimental bed in conjunction with the effective mixing of particles will arguably result in a rather narrow charge distribution among the particles: this could reasonably explain the correspondence of the experiments and the simulation results with an uniform charge distribution. This then suggests that the shortcoming of the dynamic tribocharging model can be explained to a good extent by the speed at which charge transfer is allowed to occur between the particles in our simulations. Slower charge transfer in the tribocharging model would enable a larger extent of mixing, but it would increase the computational cost of the CFD-DEM simulations beyond our current resources. Alternative simulation approaches, such as a Euler-Euler model with tribocharging, if developed, could permit slower charge transfer rates. 


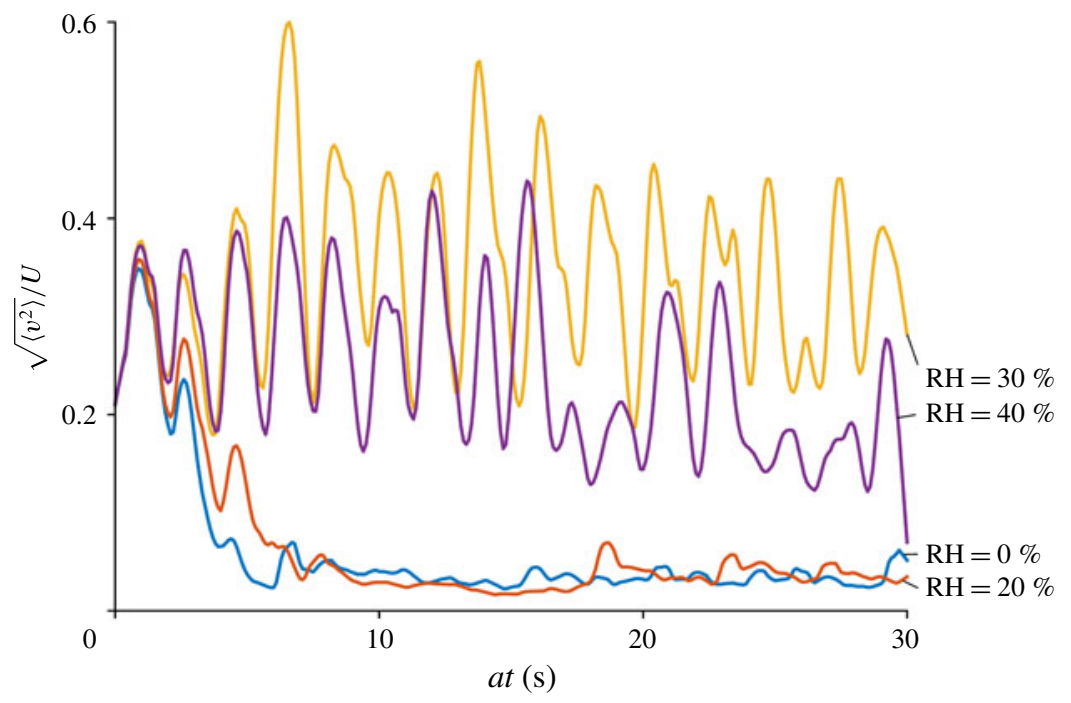

FIGURE 10. (Colour online) The root mean square speed of the simulated particles on the wall when $a=10$ (in the area corresponding to the imaged area of the experimental bed).

Although lowering the acceleration factor results in less pronounced charge segregation, even an acceleration factor as low as $a=2$ does not properly predict agglomeration of multiple layers on the wall. This is attributed to the fact that the charging model tends to over-predict the charging rate in the first place, which was also observed in our previous experiments with a vibrated bed (Kolehmainen et al. 2017b). The underlying assumptions of the surface state model may explain the discrepancy between the simulated and experimental charging rates: for example it is assumed that the particles are perfectly spherical, but in reality charge localization at the asperities of the surfaces can significantly alter the electric field in the contact gap and hence the charging rate.

Regardless of the triboelectric charging model, a notable deficiency of the present simulations is that the particles do not form a completely static structure on the wall. This is indicated by the residual r.m.s. speed shown in figure 10. In reality, sheeting of the wall may significantly limit the net charge transferred to the bed as no tribocharging occurs between the wall and a layer of motionless particles. This aspect is currently not captured by the simulations: a mobile particle will drain charge from the wall whenever the particle-wall contact area increases.

In the case of an unipolar charge distribution, the short-range electrostatic forces are repulsive and there is a lack of attractive forces between the particles. That said, attractive forces not accounted in this study could explain formation of motionless particle layers on the wall. A plausible explanation for such a structure could be a bipolar charge distribution (Salama et al. 2013; Song \& Mehrani 2017), which in single-component systems is associated with different charging tendencies of differently sized particles (Cartwright et al. 1985; Mehrani, Bi \& Grace 2005; Forward, Lacks \& Sankaran 2008). Even with tightly monodisperse particles, the effective work function could be somewhat different for the various particles (as opposed to the same value assumed in our simulations), for example, due to the differences in surface heterogeneities. This could lead to bipolar charging even for the monodisperse case (Lee et al. 2015). 

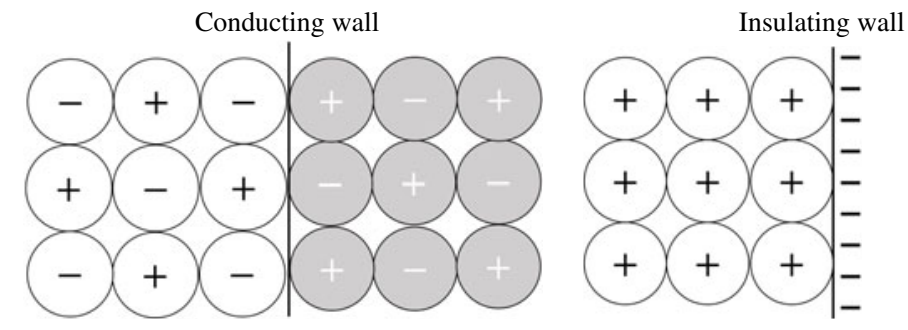

FIgURE 11. Possible structures of charged particles on a conducting and an insulating wall. The grey particles denote the image charge due to the conducting wall (Song \& Mehrani 2017).

In the case of insulating walls, the surface charge on the wall would arguably prevent oppositely charged particles from attaching on the first layer but allow adhesion of similarly charge particles as depicted in figure 11. On conducting grounded walls, however, particles with both polarities may be attracted on the wall due to the image charge effect (Song \& Mehrani 2017). Other factors such as dielectrophoresis are likely required to explain formation of a motionless particle structures: on close approach, dielectrophoretic forces can pull same-polarity particles with different charge magnitudes together (LaMarche et al. 2010; Liu et al. 2010; Lee et al. 2015; Siu et al. 2015). In addition to dielectrophoresis, polarization is likely to have a direct effect on triboelectric charging. In terms of the present charging model, polarization affects the electric field developed in the gap between two surfaces, which affects both the charging rate and the saturation charge of the particles.

\section{Conclusions}

It was found that the charge acquired by polyethylene particles in a fluidized bed made of soda-lime glass walls depends heavily on the humidity of the fluidizing gas. In relatively dry conditions the column walls become sheeted with multiple layers of particles, resulting in a significant decrease in the pressure drop across the bed. Interestingly, the polarity of the charge accumulated on the particles reverses sign at approximately $30 \%$ relative humidity. Complementary simulations were performed to examine whether a tribocharging model, tuned to capture the experimentally measured charge level, would predict the experimentally observed large variation of the pressure drop with charging extent.

A triboelectric charging model in conjunction with the CFD-DEM method was used to simulate the experimental bed. A PPPM method was used to evaluate the electric field acting on each particle while accounting the contribution of the charge on the insulating walls. It was found that artificially increasing the charge transfer rate leads to a wider charge distribution. Particles with high level of charge tend to segregate to the walls, and the remaining particles with less charges are unable to form wall layers that are multiple particle thick; furthermore, the model predicts the experimentally observed pressure drop rather poorly. When the same level of charge is included in the analysis while distributing the charges uniformly on the particles, the pressure drop in the experiment is remarkably well predicted. This suggests that the actual rate of charge transfer in the real system has to be a lot slower than what was used in our simulations, allowing ample opportunities for the charged particles to mix and achieve a narrower charge distribution. 
It is computationally unfeasible to simulate very slow charging rates using the Laurentie et al. (2013) model coupled with CFD-DEM. Alternative approaches to simulate particle charging could be Euler-Euler method (see Jalalinejad, Bi \& Grace 2016); multiphase particle in cell method (see Snider 2001); or projective integration method (see Gear \& Kevrekidis 2003). These methods avoid the need to solve the charge dynamics at the same time scale as the particle dynamics is solved and could therefore allow for more realistic charging time scales. However, to best of the authors knowledge the Laurentie et al. (2013) model has not been incorporated into any of these methods.

Based on the simulations it is apparent that the electrostatic forces largely explain the extent at which particles agglomerate on the wall when the charge distribution among the particles is narrow. In such a case the surface charge on the wall dominates the electric field, attracting particles over a large distance. Differing from the experiments, the simulated particles do not form a static structure on the wall. As tribocharging is dependent on the intensity of particle-wall contacts, this alters the net charge accumulated in the bed as well as the charge distribution.

Additional factors such as dielectrophoresis or size-dependent charging tendency of particles are probably required to explain the development of static particle structures. Analysing the effect of size dependency would require the tribocharging model that can predict it, but currently all the size specific tribocharging models in the literature are only applicable to systems that are made of same material (Duff \& Lacks 2008; Kok \& Lacks 2009; Carter \& Hartzell 2017). The authors suggest including dielectrophoresis in the current analysis a viable future avenue for research and the interested reader can find examples from the studies of Liu et al. (2010), Lee et al. (2015), Yoshimatsu et al. (2017).

\section{Acknowledgements}

The authors would like to thank the Academy of Finland Grant 289901 (Finland), the National Science Foundation Grant CBET-1601986 (United States) and ExxonMobil Research and Engineering Company for funding this research.

\section{REFERENCES}

Antypov, D. \& Elliott, J. A. 2011 On an analytical solution for the damped Hertzian spring. Europhys. Lett. 94 (5), 50004.

CARTer, D. \& Hartzell, C. 2017 Extension of discrete tribocharging models to continuous size distributions. Phys. Rev. E 95 (1), 012901.

Cartwright, P., Singh, S., Bailey, A. G. \& Rose, L. J. 1985 Electrostatic charging characteristics of polyethylene powder during pneumatic conveying. IEEE Trans. Ind. Applics. (2), 541-546.

Castle, G. S. P. 1997 Contact charging between insulators. J. Electrostat. 40 (Supplement C), $13-20$.

Cundall, P. A. \& Strack, O. D. L. 1979 A discrete numerical model for granular assemblies. Gèotechnique 29 (1), 47-65.

DufF, N. \& LACKS, D. J. 2008 Particle dynamics simulations of triboelectric charging in granular insulator systems. J. Electrostat. 66 (1-2), 51-57.

Feynman, R. P., Leighton, R. \& Sands, M. 1966 The Feynman Lectures on Physics, Vol. 2: Mainly Electromagnetism and Matter. Addison-Wesley.

FORWARD, K. M., LACKS, D. J. \& SANKARAN, R. M. 2008 Triboelectric charging of granular insulator mixtures due solely to particle-particle interactions. Ind. Engng Chem. Res. 48 (5), 2309-2314. 
Fotovat, F., Alsmari, T. A., Grace, J. R. \& BI, X. T. $2017 a$ The relationship between fluidized bed electrostatics and entrainment. Powder Technol. 316, 157-165.

Fotovat, F., Bi, X. T. \& Grace, J. R. $2017 b$ Electrostatics in gas-solid fluidized beds: a review. Chem. Engng Sci. 173, 303-334.

Fotovat, F., Grace, J. R. \& BI, X. T. 2017c Particle entrainment from gas-solid fluidized beds: conductive versus dielectric fines. AIChE J. 63 (4), 1194-1202.

Gear, C. W. \& Kevrekidis, I. G. 2003 Projective methods for stiff differential equations: problems with gaps in their eigenvalue spectrum. SIAM J. Sci. Comput. 24 (4), 1091-1106.

Grosshans, H. \& PAPAlEXANDris, M. V. $2016 a$ Evaluation of the parameters influencing electrostatic charging of powder in a pipe flow. J. Loss Prev. Process. Ind. 43 (Supplement C), 83-91.

Grosshans, H. \& Papalexandris, M. V. $2016 b$ Large eddy simulation of triboelectric charging in pneumatic powder transport. Powder Technol. 301 (Supplement C), 1008-1015.

GU, Z., WEI, W., SU, J. \& YU, C. W. 2013 The role of water content in triboelectric charging of wind-blown sand. Sci. Rep. 3, 1337.

HARPer, W. R. 1967 Contact and Frictional Electrification. Clarendon Press.

HENDRICKSON, G. 2006 Electrostatics and gas phase fluidized bed polymerization reactor wall sheeting. Chem. Engng Sci. 61 (4), 1041-1064.

JACKSON, J. D. 1999 Classical Electrodynamics, 3rd edn. Wiley.

JALALIneJad, F., BI, X. T. \& GRACE, J. R. 2012 Effect of electrostatic charges on single bubble in gas-solid fluidized beds. Intl J. Multiphase Flow 44, 15-28.

JAlalinejad, F., BI, X. T. \& GRACE, J. R. 2016 Comparison of theory with experiment for single bubbles in charged fluidized particles. Powder Technol. 290, 27-32.

JASAK, H., Jemcov, A. \& TUKOVIC, Z. 2007 Openfoam: A C++ library for complex physics simulations. In International Workshop on Coupled Methods in Numerical Dynamics, vol. 1000, pp. 1-20. IUC Dubrovnik.

Karner, S., Maier, M., LitTringer, E. \& Urbanetz, N. A. 2014 Surface roughness effects on the tribo-charging and mixing homogeneity of adhesive mixtures used in dry powder inhalers. Powder Technol. 264 (Supplement C), 544-549.

KNORR, N. 2011 Squeezing out hydrated protons: low-frictional-energy triboelectric insulator charging on a microscopic scale. AIP Adv. 1 (2), 022119.

KoK, J. F. \& LACKs, D. J. 2009 Electrification of granular systems of identical insulators. Phys. Rev. E 79 (5), 051304.

Kolehmainen, J., Ozel, A., Boyce, C. \& Sundaresan, S. $2017 a$ Triboelectric charging of monodisperse particles in fluidized beds. AIChE J. 63 (6), 1872-1891.

Kolehmainen, J., Ozel, A. \& Sundaresan, S. 2016 A hybrid approach to computing electrostatic forces in fluidized beds of charged particles. AIChE J. 62 (7), 2282-2295.

Kolehmainen, J., Sippola, P., Raitanen, O., Ozel, A., Boyce, C. M., SaArenrinne, P. \& SUNDARESAN, S. $2017 b$ Effect of humidity on triboelectric charging in a vertically vibrated granular bed: experiments and modeling. Chem. Engng Sci. 173, 363-373.

KorevaAr, M. W., Padding, J. T., Van Der Hoef, M. A.\& Kuipers, J. A. M. 2014 Integrated DEM-CFD modeling of the contact charging of pneumatically conveyed powders. Powder Technol. 258, 144-156.

Lacks, D. J. \& Sankaran, R. M. 2011 Contact electrification of insulating materials. J. Phys. D: Appl. Phys. 44 (45), 453001.

Lamarche, K. R., Muzzio, F. J., Shinbrot, T. \& Glasser, B. J. 2010 Granular flow and dielectrophoresis: the effect of electrostatic forces on adhesion and flow of dielectric granular materials. Powder Technol. 199 (2), 180-188.

Laurentie, J. C., Traoré, P. \& Dascalescu, L. 2013 Discrete element modeling of triboelectric charging of insulating materials in vibrated granular beds. J. Electrostat. 71 (6), 951-957.

Laurentie, J.-C., Traore, P., Dragan, C. \& Dascalescu, L. 2010 Numerical modeling of triboelectric charging of granular materials in vibrated beds. In Industry Applications Society Annual Meeting (IAS), 2010 IEEE, pp. 1-6. IEEE. 
LEE, L.-H. 1994 Dual mechanism for metal-polymer contact electrification. J. Electrostat. 32 (1), $1-29$.

Lee, V., Waitukaitis, S. R., Miskin, M. Z. \& Jaeger, H. M. 2015 Direct observation of particle interactions and clustering in charged granular streams. Nat. Phys. 11, 733-737.

LiaO, C.-C., Hsiau, S.-S. \& HuAng, T.-Y. 2011 The effect of vibrating conditions on the electrostatic charge in a vertical vibrating granular bed. Powder Technol. 208 (1), 1-6.

LiU, G., Marshall, J. S., LI, S. Q. \& YaO, Q. 2010 Discrete-element method for particle capture by a body in an electrostatic field. Intl J. Numer. Meth. Engng 84 (13), 1589-1612.

Lowell, J. \& Rose-Innes, A. C. 1980 Contact electrification. Adv. Phys. 29 (6), 947-1023.

Matsusaka, S., Maruyama, H., Matsuyama, T. \& Ghadiri, M. 2010 Triboelectric charging of powders: a review. Chem. Engng Sci. 65 (22), 5781-5807.

Matsuyama, T. \& Yамамото, H. 1995 Characterizing the electrostatic charging of polymer particles by impact charging experiments. Adv. Powder Technol. 6 (3), 211-220.

McCarty, L. S. \& Whitesides, G. M. 2008 Electrostatic charging due to separation of ions at interfaces: contact electrification of ionic electrets. Angew. Chem. Intl Ed. Engl. 47 (12), 2188-2207.

Mehrani, P., Bi, H. T. \& Grace, J. R. 2005 Electrostatic charge generation in gas-solid fluidized beds. J. Electrostat. 63 (2), 165-173.

Mehrani, P., Bi, H. T. \& Grace, J. R. 2007 Electrostatic behavior of different fines added to a Faraday cup fluidized bed. J. Electrostat. 65 (1), 1-10.

Mizutani, M., Yasuda, M. \& Matsusaka, S. 2015 Advanced characterization of particles triboelectrically charged by a two-stage system with vibrations and external electric fields. Adv. Powder Technol. 26 (2), 454-461.

Naik, S., Sarkar, S., Gupta, V., Hancock, B. C., Abramov, Y., Yu, W. \& Chaudhuri, B. 2015 A combined experimental and numerical approach to explore tribocharging of pharmaceutical excipients in a hopper chute assembly. Intl J. Pharmaceut. 491 (1), 58-68.

Naik, S., Sarkar, S., Hancock, B., Rowland, M., Abramov, Y., Yu, W. \& Chaudhuri, B. 2016 An experimental and numerical modeling study of tribocharging in pharmaceutical granular mixtures. Powder Technol. 297, 211-219.

Pähtz, T., Herrmann, H. J. \& Shinbrot, T. 2010 Why do particle clouds generate electric charges? Nat. Phys. 6 (5), 364-368.

PEI, C., WU, C.-Y. \& ADAMS, M. 2016 DEM-CFD analysis of contact electrification and electrostatic interactions during fluidization. Powder Technol. 304, 208-217.

RADL, S. \& SUNDARESAN, S. 2014 A drag model for filtered Euler-Lagrange simulations of clustered gas-particle suspensions. Chem. Engng Sci. 117, 416-425.

Rokkam, R. G., Sowinski, A., Fox, R. O., Mehrani, P. \& Muhle, M. E. 2013 Computational and experimental study of electrostatics in gas-solid polymerization fluidized beds. Chem. Engng Sci. 92, 146-156.

Rowe, P. N. 1961 Drag forces in a hydraulic model of a fluidized bed - part ii. Trans. Inst. Chem. Engrs 39, 175-180.

Salama, F., Sowinski, A., Atieh, K. \& Mehrani, P. 2013 Investigation of electrostatic charge distribution within the reactor wall fouling and bulk regions of a gas-solid fluidized bed. $J$. Electrostat. 71 (1), 21-27.

Schella, A., Herminghaus, S. \& Schroter, M. 2017 Influence of humidity on tribo-electric charging and segregation in shaken granular media. Soft Matt. 13, 394-401.

SCHILlER, L. \& NAUMANN, A. 1933 Uber die grundlegenden berechnungen bei der schwekraftaubereitung. Z. Verein. Deutsch. Ing. 77 (12), 318-320.

Siu, T., Cotton, J., Mattson, G. \& Shinbrot, T. 2014 Self-sustaining charging of identical colliding particles. Phys. Rev. E 89 (5), 052208.

Siu, T., Pittman, W., Cotton, J. \& Shinbrot, T. 2015 Nonlinear granular electrostatics. Granul. Matt. 17 (2), 165-175.

SNider, D. M. 2001 An incompressible three-dimensional multiphase particle-in-cell model for dense particle flows. J. Comput. Phys. 170 (2), 523-549. 
Song, D. \& Mehrani, P. 2017 Mechanism of particle build-up on gas-solid fluidization column wall due to electrostatic charge generation. Powder Technol. 316 (Supplement C), 166-170.

Sowinski, A., Mayne, A. \& Mehrani, P. 2012 Effect of fluidizing particle size on electrostatic charge generation and reactor wall fouling in gas-solid fluidized beds. Chem. Engng Sci. 71, 552-563.

Tanoue, K.-I., Tanaka, H., Kitano, H. \& Masuda, H. 2001 Numerical simulation of triboelectrification of particles in a gas-solids two-phase flow. Powder Technol. 118 (1), 121-129.

Thielicke, W. \& Stamhuis, E. J. 2014 Pivlab - towards user-friendly, affordable and accurate digital particle image velocimetry in matlab. J. Open Res. Software 2 (1), e30.

TOUKMAJI, A. Y. \& BOARD, J. A. JR. 1996 Ewald summation techniques in perspective: a survey. Comput. Phys. Commun. 95, 73-92.

Wen, C. Y. \& Yu, Y. H. 1966 Mechanics of fluidization. Chem. Engr. Prog. Sump. Series 62, 100.

Westerweel, J., Dabiri, D. \& Gharib, M. 1997 The effect of a discrete window offset on the accuracy of cross-correlation analysis of digital PIV recordings. Exp. Fluids 23 (1), 20-28.

YAnG, Y., ZI, C., HuAng, Z., WANG, J., Lungu, M., LiaO, Z., YANG, Y. \& Su, H. 2017 CFD-DEM investigation of particle elutriation with electrostatic effects in gas-solid fluidized beds. Powder Technol. 308, 422-433.

Yoshimatsu, R., Araujo, N. A. M., Shinbrot, T. \& Herrmann, H. J. 2016 Field driven charging dynamics of a fluidized granular bed. Soft Matt. 12, 6261-6267.

Yoshimatsu, R., Araújo, N. A. M., Wurm, G., Herrmann, H. J. \& Shinbrot, T. 2017 Self-charging of identical grains in the absence of an external field. Sci. Rep. 7, 39996.

Zhou, Z. Y., KuAng, S. B., CHU, K. W. \& YU, A. B. 2010 Discrete particle simulation of particle-fluid flow: model formulations and their applicability. J. Fluid Mech. 661, 482-510.

Zhou, Y. S., Wang, S., Yang, Y., Zhu, G., NiU, S., Lin, Z.-H., LiU, Y. \& Wang, Z. L. 2014 Manipulating nanoscale contact electrification by an applied electric field. Nano Lett. 14 (3), $1567-1572$. 